\title{
On the Complexity of Model Checking for Syntactically Maximal Fragments of the Interval Temporal Logic HS with Regular Expressions*
}

\author{
Laura Bozzelli Adriano Peron \\ University of Napoli "Federico II", Napoli, Italy \\ lr.bozzelli@gmail.com adrperon@unina.it \\ Alberto Molinari Angelo Montanari \\ University of Udine, Udine, Italy \\ molinari.alberto@gmail.com angelo.montanari@uniud.it
}

\begin{abstract}
In this paper, we investigate the model checking (MC) problem for Halpern and Shoham's interval temporal logic HS. In the last years, interval temporal logic $\mathrm{MC}$ has received an increasing attention as a viable alternative to the traditional (point-based) temporal logic $\mathrm{MC}$, which can be recovered as a special case. Most results have been obtained under the homogeneity assumption, that constrains a proposition letter to hold over an interval if and only if it holds over each component state. Recently, Lomuscio and Michaliszyn proposed a way to relax such an assumption by exploiting regular expressions to define the behaviour of proposition letters over intervals in terms of their component states. When homogeneity is assumed, the exact complexity of $\mathrm{MC}$ is a difficult open question for full $H S$ and for its two syntactically maximal fragments $A \bar{A} B \overline{B E}$ and $A \bar{A} E \overline{B E}$. In this paper, we provide an asymptotically optimal bound to the complexity of these two fragments under the more expressive semantic variant based on regular expressions by showing that their MC problem is $\mathbf{A E X P}_{\text {pol-complete, where }} \mathbf{A E X P}_{\text {pol }}$ denotes the complexity class of problems decided by exponential-time bounded alternating Turing Machines making a polynomially bounded number of alternations.
\end{abstract}

\section{Introduction}

Model checking (MC), which allows one to automatically check whether a model of a given system satisfies a desired behavioural property, is commonly recognized as one of the most effective techniques in automatic system verification. Besides in formal verification, it has been successfully used also in more general contexts (e.g., databases, planning, configuration systems, multi-agent systems [12, 18]). The actual possibility of exploiting MC relies on a good balance of expressiveness and complexity in the choice of the system model and of the language for specifying behavioural properties. Systems are usually modeled as finite state-transition graphs (finite Kripke structures), while properties are commonly expressed by formulas of point-based temporal logics, such as LTL, CTL, and CTL* [25, 10].

In this paper, we focus on MC with interval temporal logic (ITL) as the specification language. ITL features intervals, instead of points, as its primitive temporal entities [13, 24, 28]. ITL allows one to deal with relevant temporal properties, such as actions with duration, accomplishments, and temporal aggregations, which are inherently "interval-based" and cannot be properly expressed by point-based

${ }^{*}$ The work by Alberto Molinari and Angelo Montanari has been supported by the GNCS project Logic and Automata for Interval Model Checking.

P. Bouyer, A. Orlandini \& P. San Pietro (Eds.): 8th Symposium on Games, Automata, Logics and Formal Verification (GandALF'17) EPTCS 256, 2017, pp. 31-45 doi 10.4204/EPTCS.256.3 (c) L. Bozzelli, A. Molinari, A. Montanari \& A. Peron This work is licensed under the Creative Commons Attribution-Noncommercial-No Deriv. Works License. 
temporal logics. ITL has been fruitfully applied in various areas of computer science, including formal verification, computational linguistics, planning, and multi-agent systems [24, 26, 15].

Among ITLs, the landmark is Halpern and Shoham's modal logic of time intervals HS [13], which features one modality for each of the 13 ordering relations between pairs of intervals (the so-called Allen's relations [1]), apart from equality. (Actually, the three Allen's modalities meets A, started-by B, and finished-by $\mathrm{E}$, together with the corresponding inverse modalities $\bar{A}, \bar{B}$, and $\bar{E}$, suffice for expressing the entire set of relations.) The satisfiability problem for HS is undecidable over all relevant classes of linear orders [13], and most of its fragments (with meaningful exceptions) are undecidable as well [8, 19].

The MC problem for HS and its fragments consists in the verification of the correctness of the behaviour of a given system with respect to interval properties expressed in HS. Each finite computation path is interpreted as an interval, and its labelling is defined on the basis of the labelling of the states occurring in the path. Most results have been obtained by imposing suitable restrictions on proposition letters labeling intervals: either a proposition letter can be constrained to hold over an interval if and only if it holds over each component state (homogeneity assumption [27]), or interval labeling can be defined in terms of the labeling of interval endpoints.

An almost complete picture of the MC problem for full HS and its fragments has been recently depicted with the contribution of many works by Molinari et al. [20, 21, 22, 5, 7, 20, 23], which all consider MC over finite Kripke structures for HS endowed with a state-based semantics (allowing branching both in the past and in the future) enforcing the homogeneity assumption. The summary of these results is depicted in the second column of Table 1 (the first column reports the fragments of HS denoted by the list of the featured modalities). The complexity classes shown in red represent new (upper/lower) bounds to the complexity of the problem deriving from the results of this paper, while the other classes (in black) are known bounds. Only few, hard issues are left open in this picture, mostly regarding the precise complexity of the full logic and its maximal fragments. A comparison of different semantic solutions (i.e., state-based semantics, trace-based semantics and computation-tree-based semantics), together with an expressiveness comparison with standard point-based temporal logics LTL, CTL, and CTL* can be found in [6].

Different assumptions have been done by Lomuscio and Michaliszyn in [15, 16] for some HS fragments extended with epistemic operators $(K C)$. They assume a computation-tree-based semantics (formulae are interpreted over the unwinding of the Kripke structure) and interval labeling takes into account only the endpoints of intervals. The different semantic assumptions prevent any immediate comparison with respect to the former approach. The decidability status of MC for full epistemic HS is still unknown. (A summary of the results by Lomuscio and Michaliszyn is depicted in the last column of Table 1)

The first meaningful attempt to relax the homogeneity assumption can be found in [17], where Lomuscio and Michaliszyn propose to use regular expressions to define the labeling of proposition letters over intervals in terms of the component states. Note that the homogeneity assumption can be trivially encoded by regular expressions. In that work, the authors prove the decidability of MC with regular expressions for some very restricted fragments of epistemic HS, giving some rough upper bounds to its computational complexity. A deeper insight into the problem of MC for HS with regular expressions can be found in [3] where, under the assumption of a state-based semantics, it is proved that MC with regular expressions for full HS is decidable, and that a large class of HS fragments can be checked in polynomial working space (see the third column of Table 1).

In this paper, we study the problems of $\mathrm{MC}$ for the two (syntactically) maximal (symmetric) fragments $A \bar{A} B \overline{B E}$ and $A \bar{A} E \overline{B E}$ with regular expressions, which are not covered by [3], proving that the complexity of both problems is $\mathbf{A E X P}_{\text {pol-complete. }} \mathbf{A E X P}_{\text {pol }}$ denotes the complexity class of problems decided by exponential-time bounded alternating Turing Machines with a polynomially bounded number 
Table 1: Complexity of MC for HS and its fragments ( ${ }^{\dagger}$ local MC).

\begin{tabular}{|c|c|c|c|}
\hline & Homogeneity & Regular expressions & {$[15]-[17]$} \\
\hline Full HS, BE & $\begin{array}{c}\text { non-elem. } \\
\text { EXPSPACE-hard }\end{array}$ & $\begin{array}{c}\text { non-elem. } \\
\text { EXPSPACE-hard }\end{array}$ & $\begin{array}{c}\mathrm{BE}+K C^{\dagger}: \text { PSPACE } \\
\mathrm{BE}^{\dagger}: \mathbf{P}\end{array}$ \\
\hline $\mathrm{A} \overline{\mathrm{A}} \mathrm{B} \overline{\mathrm{BE}}, \mathrm{A} \overline{\mathrm{A}} \mathrm{E} \overline{\mathrm{BE}}$ & $\begin{array}{c}\in \text { EXPSPACE }\left[\in \text { AEXP }_{\text {pol }}\right] \\
\text { PSPACE-hard }\end{array}$ & $\begin{array}{c}\text { non-elem PSPACE-hard } \\
\text { [AEXP } \mathbf{p}_{\text {pol-complete }}\end{array}$ & \\
\hline $\mathrm{A} \overline{\mathrm{ABE}}$ & PSPACE-complete & $\begin{array}{c}\text { non-elem }\left[\in \mathbf{A E X P}_{\text {pol }}\right] \\
\text { PSPACE-hard }\end{array}$ & \\
\hline $\begin{array}{l}\mathrm{A} \overline{\mathrm{A}} \mathrm{B} \overline{\mathrm{B}}, \mathrm{B} \overline{\mathrm{B}}, \overline{\mathrm{B}} \\
\mathrm{A} \overline{\mathrm{A}} \mathrm{E} \overline{\mathrm{E}}, \mathrm{E} \overline{\mathrm{E}}, \overline{\mathrm{E}}\end{array}$ & PSPACE-complete & PSPACE-complete & $\mathrm{A} \overline{\mathrm{B}}+K C:$ non-elem. \\
\hline $\mathrm{A} \overline{\mathrm{A}} \mathrm{B}, \mathrm{A} \overline{\mathrm{A}} \mathrm{E}, \mathrm{AB}, \overline{\mathrm{A}} \mathrm{E}$ & $\mathbf{P}^{\mathbf{N P}}$-complete & PSPACE-complete & \\
\hline $\mathrm{A} \overline{\mathrm{A}}, \overline{\mathrm{A}} \mathrm{B}, \mathrm{AE}, \mathrm{A}, \overline{\mathrm{A}}$ & $\begin{array}{c}\in \mathbf{P}^{\mathbf{N P}\left[O\left(\log ^{2} n\right)\right]} \\
\mathbf{P}^{\mathbf{N P}[O(\log n)]_{\text {-hard }}}\end{array}$ & PSPACE-complete & \\
\hline Prop, B, E & co-NP-complete & PSPACE-complete & \\
\hline
\end{tabular}

of alternations. Such a class captures the precise complexity of some relevant problems [2, 11] (e.g., the first-order theory of real addition with order [11]). First, we note that settling the exact complexity of these fragments under the homogeneity assumption (which can be encoded by regular expressions) is a difficult open question [22]. Moreover, considering that AEXP $_{\text {pol }} \subseteq$ EXPSPACE and that HS under homogeneity is subsumed by HS with regular expressions, the results proved in this paper improve the upper bounds for the fragments $A \bar{A} B \overline{B E}$ and $A \bar{A} E \overline{B E}$ given in [22].

These results are obtained by preliminarily establishing an exponential-size model-trace property: for each interval, it is possible to find an interval of bounded exponential length that is indistinguishable with respect to the fulfillment of $A \bar{A} B \overline{B E}$ formulas (resp., $A \bar{A} E \overline{B E}$ ). Such a property allows us to devise a MC procedure belonging to the class $\mathbf{A E X P}_{\text {pol }}$. Finally, the matching lower bounds are obtained by polynomial-time reductions from the so-called alternating multi-tiling problem, and they already hold for the fragments $B \bar{E}$ and $E \bar{B}$ of $A \bar{A} B \overline{B E}$ and $A \bar{A} E \overline{B E}$, respectively.

The paper is structured as follows. In Section 2, we introduce the logic HS and provide some background knowledge. In Section 3 we prove the exponential-size model-trace property for $A \bar{A} B \overline{B E}$. In Section 4, we provide an $\mathbf{A E X P}_{\text {pol }}$ upper bound to the $\mathrm{MC}$ problem for $A \bar{A} B \overline{B E}$. Finally, in Section 5 . we prove the hardness of the fragment $B \bar{E}$. Similar proofs can be given for establishing the $\mathbf{A E X P}_{\mathbf{p o l}^{-}}$ completeness of $A \bar{A} E \overline{B E}$, and the $\mathbf{A E X P}_{\text {pol }}$-hardness of $E \bar{B}$.

Due to space constraints, most of the proofs are omitted here: they can be found in [4].

\section{Preliminaries}

We introduce some preliminary notation. Let $\mathbb{N}$ be the set of natural numbers. For all $i, j \in \mathbb{N}$, with $i \leq j$, $[i, j]$ denotes the set of natural numbers $h$ such that $i \leq h \leq j$.

Let $\Sigma$ be an alphabet and $w$ be a finite word over $\Sigma$. We denote by $|w|$ the length of $w$. By $\varepsilon$ we denote the empty word. For all $1 \leq i \leq j \leq|w|, w(i)$ denotes the $i$-th letter of $w$, while $w(i, j)$ 
denotes the finite subword of $w$ given by $w(i) w(i+1) \cdots w(j)$. For $|w|=n$, we define fst $(w)=w(1)$ and lst $(w)=w(n)$. The sets of all proper prefixes and suffixes of $w$ are $\operatorname{Pref}(w)=\{w(1, i) \mid 1 \leq i \leq n-1\}$ and $\operatorname{Suff}(w)=\{w(i, n) \mid 2 \leq i \leq n\}$, respectively. The concatenation of two words $w$ and $w^{\prime}$ is denoted as usual by $w \cdot w^{\prime}$. Moreover, if $1 \operatorname{st}(w)=\operatorname{fst}\left(w^{\prime}\right), w \star w^{\prime}$ represents $w(1, n-1) \cdot w^{\prime}$, where $n=|w|(\star-$ concatenation).

\subsection{Kripke structures, regular expressions, and finite automata}

Finite state systems are usually modelled as finite Kripke structures. Let $\mathcal{A P}$ be a finite set of proposition letters, which represent predicates decorating the states of the given system.

Definition 1 (Kripke structure). A Kripke structure over $\mathcal{A P}$ is a tuple $\mathcal{K}=\left(\mathcal{A P}, S, R, \mu, s_{0}\right)$, where $S$ is a set of states, $R \subseteq S \times S$ is a transition relation, $\mu: S \mapsto 2^{\mathscr{A P} P}$ is a total labelling function assigning to each state $s$ the set of propositions that hold over it, and $s_{0} \in S$ is the initial state. $\mathcal{K}$ is said finite if $S$ is finite.

Let $\mathcal{K}=\left(\mathscr{A P}, S, R, \mu, s_{0}\right)$ be a Kripke structure. A trace (or finite path) of $\mathcal{K}$ is a non-empty finite word $\rho$ over $S$ such that $(\rho(i), \rho(i+1)) \in R$ for all $i \in[1,|\rho|-1]$. A trace is initial if it starts from the initial state $s_{0}$. A trace $\rho$ induces the finite word $\mu(\rho)$ over $2^{\mathscr{A P}}$ given by $\mu(\rho(1)) \cdots \mu(\rho(n))$ with $n=|\rho|$. We call $\mu(\rho)$ the labeling sequence induced by $\rho$.

Let us recall now the class of regular expressions over finite words. Since we are interested in expressing requirements over the labeling sequences induced by the traces of Kripke structures, which are finite words over $2^{\mathfrak{A P}}$, here we consider propositional-based regular expressions (RE), where the atomic expressions are propositional formulas over $\mathcal{A P}$ instead of letters over an alphabet. Formally, the set of RE $r$ over $\mathcal{A P}$ is defined as

$$
r::=\varepsilon|\phi| r \cup r|r \cdot r| r^{*}
$$

where $\phi$ is a propositional formula over $\mathcal{A P}$. The size $|r|$ of an RE $r$ is the number of subexpressions of $r$. An RE $r$ denotes a language $\mathscr{L}(r)$ of finite words over $2^{\mathscr{A P}}$ defined as:

- $\mathscr{L}(\varepsilon)=\{\varepsilon\}$

- $\mathscr{L}(\phi)=\left\{A \in 2^{\mathscr{A P}} \mid A\right.$ satisfies $\left.\phi\right\}$;

- $\mathscr{L}\left(r_{1} \cup r_{2}\right)=\mathscr{L}\left(r_{1}\right) \cup \mathscr{L}\left(r_{2}\right)$;

- $\mathscr{L}\left(r_{1} \cdot r_{2}\right)=\mathscr{L}\left(r_{1}\right) \cdot \mathscr{L}\left(r_{2}\right)$;

- $\mathscr{L}\left(r^{*}\right)=(\mathscr{L}(r))^{*}$.

We also recall the class of nondeterministic finite automata over finite words (NFA). An NFA is a tuple $\mathscr{A}=\left(\Sigma, Q, Q_{0}, \Delta, F\right)$, where $\Sigma$ is a finite alphabet, $Q$ is a finite set of states, $Q_{0} \subseteq Q$ is the set of initial states, $\Delta \subseteq Q \times \Sigma \times Q$ is the transition relation, and $F \subseteq Q$ is the set of accepting states. An NFA $\mathscr{A}$ is complete if, for all $(q, \sigma) \in Q \times \Sigma,\left(q, \sigma, q^{\prime}\right) \in \Delta$ for some $q^{\prime} \in Q$. Given a finite word $w$ over $\Sigma$ with $|w|=n$ and two states $q, q^{\prime} \in Q$, a run of $\mathscr{A}$ from $q$ to $q^{\prime}$ over $w$ is a sequence of states $q_{1}, \ldots, q_{n+1}$ such that $q_{1}=q, q_{n+1}=q^{\prime}$, and for all $i \in[1, n],\left(q_{i}, w(i), q_{i+1}\right) \in \Delta$. The language $\mathscr{L}(\mathscr{A})$ accepted by $\mathscr{A}$ is the set of finite words $w$ on $\Sigma$ such that there is a run from some initial state to some accepting state over $w$.

Remark 2. Given a RE $r$, by a standard construction [14], one can compositionally construct a complete NFA $\mathscr{A}_{r}$ with alphabet $2^{\mathscr{A P}}$, whose number of states is linear in the size of $r$. We call $\mathscr{A}_{r}$ the canonical NFA associated with $r$.

\subsection{The interval temporal logic HS}

A systematic logical study of interval representation and reasoning was proposed by J. Y. Halpern and Y. Shoham, who introduced the interval temporal logic HS [13] featuring one modality for each Allen 
Table 2: Allen's relations and corresponding HS modalities.

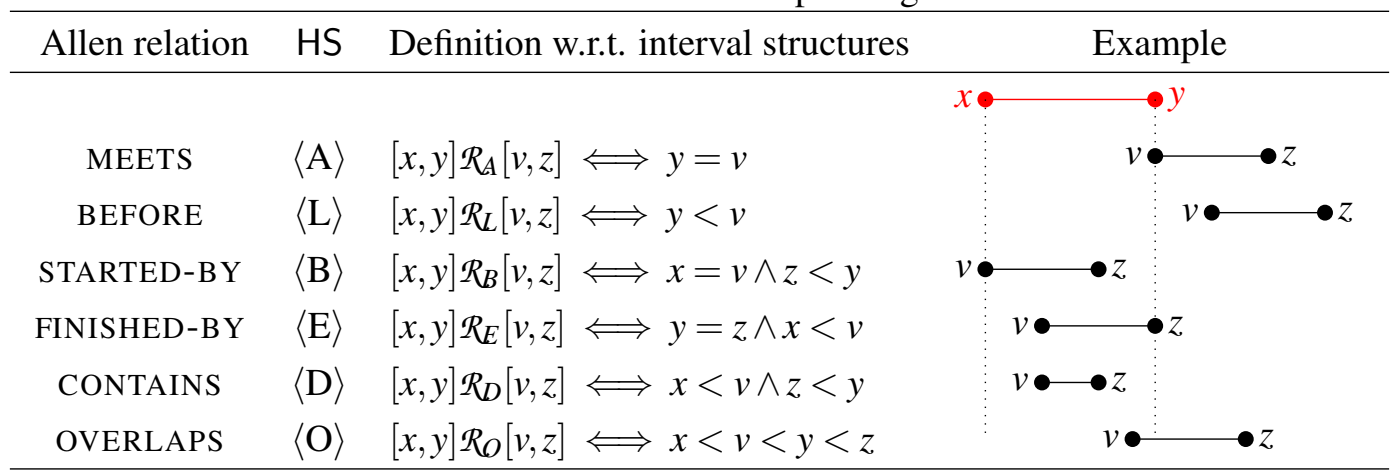

relation [1], but equality. Table 2 depicts 6 of the 13 Allen's relations, together with the corresponding HS (existential) modalities. The other 7 relations are the 6 inverse relations (given a binary relation $\mathcal{R}$, its inverse $\overline{\mathcal{R}}$ is such that $b \overline{\mathcal{R}} a$ iff $a \mathcal{R} b$ ) and equality.

Given a finite set $\mathcal{P}_{u}$ of uninterpreted interval properties, the HS language over $\mathcal{P}_{u}$ consists of propositions from $\mathscr{P}_{u}$, the Boolean connectives $\neg$ and $\wedge$, and a temporal modality for each of the (non trivial) Allen's relations, i.e., $\langle\mathrm{A}\rangle,\langle\mathrm{L}\rangle,\langle\mathrm{B}\rangle,\langle\mathrm{E}\rangle,\langle\mathrm{D}\rangle,\langle\mathrm{O}\rangle,\langle\overline{\mathrm{A}}\rangle,\langle\overline{\mathrm{L}}\rangle,\langle\overline{\mathrm{B}}\rangle,\langle\overline{\mathrm{E}}\rangle,\langle\overline{\mathrm{D}}\rangle$, and $\langle\overline{\mathrm{O}}\rangle$. HS formulas are defined by the grammar

$$
\psi::=p_{u}|\neg \psi| \psi \wedge \psi \mid\langle X\rangle \psi
$$

where $p_{u} \in \mathcal{P}_{u}$ and $X \in\{A, L, B, E, D, O, \bar{A}, \bar{L}, \bar{B}, \bar{E}, \bar{D}, \bar{O}\}$. We also exploit the standard logical connectives (disjunction $\vee$ and implication $\rightarrow$ ) as abbreviations. Furthermore, for any existential modality $\langle X\rangle$, the dual universal modality $[X] \psi$ is defined as $\neg\langle X\rangle \neg \psi$.

An HS formula $\varphi$ is in positive normal form $(P N F)$ if negation is applied only to atomic formulas in $\mathcal{P}_{u}$. By using De Morgan's laws and for any existential modality $\langle X\rangle$, the dual universal modality $[X]$, we can convert in linear-time an HS formula $\varphi$ into an equivalent formula in $P N F$, called the $P N F$ of $\varphi$. For a formula $\varphi$ in $P N F$, the dual $\widetilde{\varphi}$ of $\varphi$ is the $P N F$ of $\neg \varphi$.

Given any subset of Allen's relations $\left\{X_{1}, \ldots, X_{n}\right\}$, we denote by $X_{1} \cdots X_{n}$ the HS fragment closed under Boolean connectives that features (existential and universal) modalities for $X_{1}, \ldots, X_{n}$ only.

Without loss of generality, we assume the non-strict semantics of HS, which admits intervals consisting of a single point. (All the results we prove in the paper hold for the strict semantics as well.) Under such an assumption, all HS modalities can be expressed in terms of modalities $\langle\mathrm{B}\rangle,\langle\mathrm{E}\rangle,\langle\overline{\mathrm{B}}\rangle$, and $\langle\overline{\mathrm{E}}\rangle[28]$. HS can, thus, be viewed as a multi-modal logic with 4 primitive modalities. However, since we focus on the HS fragments $A \bar{A} E \overline{B E}$ and $A \bar{A} B \overline{B E}$, that do not feature $\langle B\rangle$ and $\langle E\rangle$ respectively, we also consider the modalities $\langle\mathrm{A}\rangle$ and $\langle\overline{\mathrm{A}}\rangle$. Note that the modalities $\langle\mathrm{L}\rangle$ and $\langle\mathrm{O}\rangle$ (resp., $\langle\overline{\mathrm{L}}\rangle$ and $\langle\overline{\mathrm{O}}\rangle$ ) can be expressed in the fragment $A \bar{A} E \overline{B E}$ (resp., $A \bar{A} B \overline{B E}$ ).

As for the semantics of HS, in this paper we follow the approach of [3], where the intervals correspond to the traces of a finite Kripke structure $\mathcal{K}$ (state-based semantics) and each abstract interval proposition $p_{u} \in \mathcal{P}_{u}$ denotes a regular language of finite words over $2^{\mathfrak{A P}}$. More specifically, every abstract interval proposition $p_{u}$ is a (propositional-based) regular expression over $\mathscr{A} \mathcal{P}$. Thus, in the following, for the sake of simplicity, by an HS formula over $\mathscr{A P}$ we mean an HS formula whose abstract interval propositions (or atomic formulas) are RE over $\mathcal{A} P$.

Given a Kripke structure $\mathcal{K}=\left(\mathscr{A P}, S, E, \mu, s_{0}\right)$ over $\mathcal{A P}$, a trace $\rho$ of $\mathcal{K}$, and an HS formula $\varphi$ over $\mathcal{A P}$, the satisfaction relation $\mathcal{K}, \rho \models \varphi$ is inductively defined as follows (we omit the standard clauses for 
the Boolean connectives):

$$
\begin{array}{ll}
\mathcal{K}, \rho=r & \Leftrightarrow \mu(\rho) \in \mathscr{L}(r) \text { for each RE } r \text { over } \mathcal{A P}, \\
\mathcal{K}, \rho=\langle\mathrm{B}\rangle \varphi & \Leftrightarrow \text { there exists } \rho^{\prime} \in \operatorname{Pref}(\rho) \text { such that } \mathcal{K}, \rho^{\prime}=\varphi, \\
\mathcal{K}, \rho=\langle\mathrm{E}\rangle \varphi & \Leftrightarrow \text { there exists } \rho^{\prime} \in \operatorname{Suff}(\rho) \text { such that } \mathcal{K}, \rho^{\prime} \models \varphi, \\
\mathcal{K}, \rho=\langle\overline{\mathrm{B}}\rangle \varphi & \Leftrightarrow \mathcal{K}, \rho^{\prime} \models \varphi \text { for some trace } \rho^{\prime} \text { such that } \rho \in \operatorname{Pref}\left(\rho^{\prime}\right), \\
\mathcal{K}, \rho=\langle\overline{\mathrm{E}}\rangle \varphi & \Leftrightarrow \mathcal{K}, \rho^{\prime} \models \varphi \text { for some trace } \rho^{\prime} \text { such that } \rho \in \operatorname{Suff}\left(\rho^{\prime}\right), \\
\mathcal{K}, \rho=\langle\mathrm{A}\rangle \varphi & \Leftrightarrow \mathcal{K}, \rho^{\prime} \models \varphi \text { for some trace } \rho^{\prime} \text { such that } \operatorname{fst}\left(\rho^{\prime}\right)=\operatorname{lst}(\rho), \\
\mathcal{K}, \rho=\langle\overline{\mathrm{A}}\rangle \varphi & \Leftrightarrow \mathcal{K}, \rho^{\prime} \models \varphi \text { for some trace } \rho^{\prime} \text { such that } \operatorname{lst}\left(\rho^{\prime}\right)=\operatorname{fst}(\rho) .
\end{array}
$$

$\mathcal{K}$ is a model of $\varphi$, denoted $\mathcal{K} \models \varphi$, if for all initial traces $\rho$ of $\mathcal{K}$, it holds that $\mathcal{K}, \rho \models \varphi$. The MC problem for HS is checking, for a finite Kripke structure $\mathcal{K}$ and an HS formula $\varphi$, whether $\mathcal{K}=\varphi$ or not.

Note that the state-based semantics provides a branching-time setting both in the past and in the future. In particular, while the modalities for $B$ and $E$ are linear-time (they allow us to select prefixes and suffixes of the current trace), the modalities for $A$ and $\bar{B}$ (resp., $\bar{A}$ and $\bar{E}$ ) are branching-time in the future (resp., in the past) since they allow us to nondeterministically extend a trace in the future (resp., in the past). As shown in [6], for the considered semantics, the logics HS and CTL* are expressively incomparable already under the homogeneity assumption. However, under the homogeneity assumption, the use of the past branching-time modalities $\bar{A}$ and $\bar{E}$ is necessary for capturing requirements which cannot be expressed in CTL*. For instance, the requirement "each state reachable from the initial one where $p$ holds has a predecessor where p holds as well" cannot be expressed in CTL*, but can be easily expressed in the fragment $\bar{A} E[\overline{6}]$. In the more expressive setting based on regular expressions, the future branching-time modalities $A$ and $\bar{B}$ are already sufficient for capturing requirements which cannot be expressed in $\mathrm{CTL}^{*}$, such as the following branching-time bounded response property: "for each state reachable from the initial one where a request req occurs, there is a computation from this state such that the request is followed by a response res within an even number of steps". This requirement can be expressed in the fragment $\mathrm{A} \overline{\mathrm{B}}$ as follows: $[\mathrm{A}]\left(\mathrm{req} \rightarrow\langle\overline{\mathrm{B}}\rangle\left(\mathrm{req} \cdot(\top \cdot T)^{*} \cdot\right.\right.$ res $\left.)\right)$.

In the rest of the paper, we focus on the fragment $A \bar{A} B \overline{B E}$. Analogous constructions and results can be symmetrically given for the fragment $A \bar{A} E \overline{B E}$ as well.

\section{Exponential-size model-trace property for $A \bar{A} B \overline{B E}$}

In this section, we show an exponential-size model-trace property for $A \bar{A} B \overline{B E}$, which will be used as the basic step to prove that the MC problem for $A \bar{A} B \overline{B E}$ belongs to $\mathbf{A E X P}_{\mathbf{p o l}}$. Fix a Kripke structure $\mathcal{K}=\left(\mathcal{A P}, S, R, \mu, s_{0}\right)$ and a finite set spec $=\left\{r_{1}, \ldots, r_{H}\right\}$ of (propositional-based) regular expressions over $\mathcal{A P}$ : such a property ensures that for each $h \geq 0$ and trace $\rho$ of $\mathcal{K}$, it is possible to build another trace $\rho^{\prime}$ of $\mathcal{K}$, of bounded exponential length, which is indistinguishable from $\rho$ with respect to the fulfilment of any $\mathrm{A} \overline{\mathrm{A}} \mathrm{B} \overline{\mathrm{B} E}$ formula $\varphi$ having atomic formulas in spec and nesting depth of the modality $\langle\mathrm{B}\rangle$ at most $h$ (written $\mathrm{d}_{\mathrm{B}}(\varphi) \leq h$ ). Formally, $\mathrm{d}_{\mathrm{B}}(\varphi)$ is inductively defined as follows $(i) \mathrm{d}_{\mathrm{B}}(r)=0$, for any $\mathrm{RE} r$ over $\mathcal{A P} ;(i i) \mathrm{d}_{\mathrm{B}}(\neg \psi)=\mathrm{d}_{\mathrm{B}}(\psi) ;(i i i) \mathrm{d}_{\mathrm{B}}(\psi \wedge \phi)=\max \left\{\mathrm{d}_{\mathrm{B}}(\psi), \mathrm{d}_{\mathrm{B}}(\phi)\right\} ;(i v) \mathrm{d}_{\mathrm{B}}(\langle\mathrm{B}\rangle \psi)=1+\mathrm{d}_{\mathrm{B}}(\psi)$; (v) $\mathrm{d}_{\mathrm{B}}(\langle\mathrm{X}\rangle \psi)=\mathrm{d}_{\mathrm{B}}(\psi)$, for $X \in\{A, \bar{A}, \bar{B}, \bar{E}\}$.

In order to state the result, we first introduce the notion of $h$-prefix bisimilarity between a pair of traces $\rho$ and $\rho^{\prime}$ of $\mathcal{K}$. As proved by Proposition 8 below, $h$-prefix bisimilarity is a sufficient condition for two traces $\rho$ and $\rho^{\prime}$ to be indistinguishable with respect to the fulfillment of any A $\bar{A} B \overline{B E}$ formula $\varphi$ over spec with $\mathrm{d}_{\mathrm{B}}(\varphi) \leq h$. Then, for a given trace $\rho$, we show how to determine a subset of positions of $\rho$, 
called the $h$-prefix sampling of $\rho$, that allows us to build another trace $\rho^{\prime}$ having singly exponential length (both in $h$ and $\mid$ spec $\mid$, where $\mid$ spec $\mid$ is defined as $\sum_{r \in \text { spec }}|r|$ ) such that $\rho$ and $\rho^{\prime}$ are $h$-prefix bisimilar.

For any regular expression $r_{\ell}$ in spec with $\ell \in[1, H]$, let $\mathscr{A}_{\ell}=\left(2^{\mathscr{A P}}, Q_{\ell}, Q_{\ell}^{0}, \Delta_{\ell}, F_{\ell}\right)$ be the canonical (complete) NFA accepting $\mathscr{L}\left(r_{\ell}\right)$ (recall that $\left|Q_{\ell}\right| \leq 2\left|r_{\ell}\right|$ ). Without loss of generality, we assume that the sets of states of these automata are pairwise disjoint.

The notion of prefix bisimilarity exploits the notion of summary of a trace $\rho$ of $\mathcal{K}$, namely a tuple "recording" the initial and final states of $\rho$, and, for each automaton $\mathscr{A}_{\ell}$ with $\ell \in[1, H]$, the pairs of states $q, q^{\prime} \in Q_{\ell}$ such that some run of $\mathscr{A}_{\ell}$ over $\mu(\rho)$ goes from $q$ to $q^{\prime}$.

Definition 3 (Summary of a trace). Let $\rho$ be a trace of $\mathcal{K}$ with $|\rho|=n$. The summary $\mathscr{S}(\rho)$ of $\rho$ (w.r.t. spec) is the triple $(\rho(1), \Pi, \rho(n))$, where $\Pi$ is the set of pairs $\left(q, q^{\prime}\right)$ such that there is $\ell \in[1, H]$ so that $q, q^{\prime} \in Q_{\ell}$ and there is a run of $\mathscr{A}_{\ell}$ from $q$ to $q^{\prime}$ over $\mu(\rho)$.

Note that the number of summaries is at most $|S|^{2} \cdot 2^{(2|\mathrm{spec}|)^{2}}$. Evidently, the following holds.

Proposition 4. Let $h \geq 0$, and $\rho$ and $\rho^{\prime}$ be two traces of $\mathcal{K}$ such that $\mathscr{S}(\rho)=\mathscr{S}\left(\rho^{\prime}\right)$. Then, for all regular expressions $r \in$ spec and traces $\rho_{L}$ and $\rho_{R}$ of $\mathcal{K}$ such that $\rho_{L} \star \rho$ and $\rho \star \rho_{R}$ are defined, the following hold: (1) $\mu(\rho) \in \mathscr{L}(r)$ iff $\mu\left(\rho^{\prime}\right) \in \mathscr{L}(r)$; (2) $\mathscr{S}\left(\rho_{L} \star \rho\right)=\mathscr{S}\left(\rho_{L} \star \rho^{\prime}\right)$; (3) $\mathscr{S}\left(\rho \star \rho_{R}\right)=\mathscr{S}\left(\rho^{\prime} \star \rho_{R}\right)$.

We now introduce the notion of prefix bisimilarity between a pair of traces $\rho$ and $\rho^{\prime}$ of $\mathcal{K}$.

Definition 5 (Prefix bisimilarity). Let $h \geq 0$. Two traces $\rho$ and $\rho^{\prime}$ of $\mathcal{K}$ are $h$-prefix bisimilar (w.r.t. spec) if the following conditions inductively hold:

- for $h=0: \mathscr{S}(\rho)=\mathscr{S}\left(\rho^{\prime}\right)$;

- for $h>0: \mathscr{S}(\rho)=\mathscr{S}\left(\rho^{\prime}\right)$ and for each proper prefix $v$ of $\rho$ (resp., proper prefix $v^{\prime}$ of $\rho^{\prime}$ ), there is a proper prefix $v^{\prime}$ of $\rho^{\prime}$ (resp., proper prefix $v$ of $\rho$ ) such that $v$ and $v^{\prime}$ are $(h-1)$-prefix bisimilar.

Property 6. For all $h \geq 0, h$-prefix bisimilarity is an equivalence relation over traces of $\mathcal{K}$.

The $h$-prefix bisimilarity of two traces $\rho$ and $\rho^{\prime}$ is preserved by right (resp., left) $\star$-concatenation with another trace of $\mathcal{K}$.

Proposition 7. Let $h \geq 0$, and $\rho$ and $\rho^{\prime}$ be two h-prefix bisimilar traces of $\mathcal{K}$. Then, for all traces $\rho_{L}$ and $\rho_{R}$ of $\mathcal{K}$ such that $\rho_{L} \star \rho$ and $\rho \star \rho_{R}$ are defined, the following hold:

(1) $\rho_{L} \star \rho$ and $\rho_{L} \star \rho^{\prime}$ are h-prefix bisimilar; (2) $\rho \star \rho_{R}$ and $\rho^{\prime} \star \rho_{R}$ are h-prefix bisimilar.

By exploiting Propositions 4 and 7, we can prove that $h$-prefix bisimilarity preserves the fulfillment of $A \bar{A} B \bar{B} E$ formulas over spec having nesting depth of modality $\langle\mathrm{B}\rangle$ at most $h$.

Proposition 8. Let $h \geq 0$, and $\rho$ and $\rho^{\prime}$ be two h-prefix bisimilar traces of $\mathcal{K}$. Then, for each $\mathrm{A} \overline{\mathrm{A}} \mathrm{B} \overline{\mathrm{BE}}$ formula $\psi$ over spec with $\mathrm{d}_{\mathrm{B}}(\psi) \leq h$, we have $\mathcal{K}, \rho \mid \psi$ iff $\mathcal{K}, \rho^{\prime} \models \psi$.

Proof. We prove the proposition by a nested induction on the structure of the formula $\psi$ and on the nesting depth $\mathrm{d}_{\mathrm{B}}(\psi)$. For the base case, $\psi$ is a regular expression in spec. Since $\mathscr{S}(\rho)=\mathscr{S}\left(\rho^{\prime}\right)(\rho$ and $\rho^{\prime}$ are $h$-prefix bisimilar) the result follows by Proposition 4. Now, let us consider the inductive case. The cases where the root modality of $\psi$ is a Boolean connective directly follow by the inductive hypothesis. As for the cases where the root modality is either $\langle\mathrm{A}\rangle$ or $\langle\overline{\mathrm{A}}\rangle$, the result follows from the fact that, being $\rho$ and $\rho^{\prime} h$-prefix bisimilar, $\operatorname{fst}(\rho)=\operatorname{fst}\left(\rho^{\prime}\right)$ and $\operatorname{lst}(\rho)=\operatorname{lst}\left(\rho^{\prime}\right)$. It remains to consider the cases where the root modality is in $\{\langle\mathrm{B}\rangle,\langle\overline{\mathrm{B}}\rangle,\langle\overline{\mathrm{E}}\rangle\}$. We prove the implication $\mathcal{K}, \rho \models \psi \Rightarrow \mathcal{K}, \rho^{\prime} \models \psi$ (the converse implication being similar). Let $\mathcal{K}, \rho=\psi$.

- $\psi=\langle\mathrm{B}\rangle \varphi$ : since $0<\mathrm{d}_{\mathrm{B}}(\psi) \leq h$, it holds that $h>0$. Since $\mathcal{K}, \rho \models\langle\mathrm{B}\rangle \varphi$, there is a proper prefix $v$ of $\rho$ such that $\mathcal{K}, v \mid=\varphi$. Since $\rho$ and $\rho^{\prime}$ are $h$-prefix bisimilar, there is a proper prefix $v^{\prime}$ of $\rho^{\prime}$ such that $v$ and $v^{\prime}$ are $(h-1)$-prefix bisimilar. Being $\mathrm{d}_{\mathrm{B}}(\varphi) \leq h-1$, by the inductive hypothesis we obtain that $\mathcal{K}, v^{\prime} \models \varphi$. Hence, $\mathcal{K}, \rho^{\prime} \models\langle\mathrm{B}\rangle \varphi$ : the thesis follows. 
- $\psi=\langle\overline{\mathrm{B}}\rangle \varphi$ : since $\mathcal{K}, \rho \models\langle\overline{\mathrm{B}}\rangle \varphi$, there is a trace $\rho_{R}$ such that $\left|\rho_{R}\right|>1$ and $\mathcal{K}, \rho \star \rho_{R} \mid=\varphi$. By Proposition 7, $\rho \star \rho_{R}$ and $\rho^{\prime} \star \rho_{R}$ are $h$-prefix bisimilar. By the inductive hypothesis on the structure of the formula, we obtain that $\mathcal{K}, \rho^{\prime} \star \rho_{R}=\varphi$, hence, $\mathcal{K}, \rho^{\prime} \models\langle\overline{\mathrm{B}}\rangle \varphi$.

- $\psi=\langle\overline{\mathrm{E}}\rangle \varphi$ : this case is similar to the previous one.

In the following, we show how a trace $\rho$, whose length exceeds a suitable exponential boundprecisely, $\left(|S| \cdot 2^{(2|\mathrm{spec}|)^{2}}\right)^{h+2}$ — can be contracted preserving $h$-prefix bisimilarity and, consequently, the fulfillment of formulas $\varphi$ with $\mathrm{d}_{\mathrm{B}}(\varphi) \leq h$. The basic contraction step of $\rho$ is performed by choosing a subset of $\rho$-positions called $h$-prefix sampling $\left(P S_{h}\right)$. A contraction can be performed whenever there are two positions $\ell<\ell^{\prime}$ satisfying $\mathscr{S}(\rho(1, \ell))=\mathscr{S}\left(\rho\left(1, \ell^{\prime}\right)\right)$ in between two consecutive positions in the linear ordering of $P S_{h}$. We prove that by taking the contraction $\rho^{\prime}=\rho(1, \ell) \cdot \rho\left(\ell^{\prime}+1,|\rho|\right)$, we obtain a trace of $\mathcal{K}$ which is $h$-prefix bisimilar to $\rho$. The basic contraction step can then be iterated over $\rho^{\prime}$ until the length bound is reached.

The notion of $h$-prefix sampling is inductively defined using the notion of prefix-skeleton sampling. For a set $I$ of natural numbers, by "two consecutive elements of $I$ " we refer to a pair of elements $i, j \in I$ such that $i<j$ and $I \cap[i, j]=\{i, j\}$.

Definition 9 (Prefix-skeleton sampling). Let $\rho$ be a trace of $\mathcal{K}$. Given two $\rho$-positions $i$ and $j$, with $i \leq j$, the prefix-skeleton sampling of $\rho$ in the interval $[i, j]$ is the minimal set $P o s \supseteq\{i, j\}$ of $\rho$-positions in the interval $[i, j]$ satisfying the condition:

- for each $k \in[i+1, j-1]$, the minimal position $k^{\prime} \in[i+1, j-1]$ such that $\mathscr{S}\left(\rho\left(1, k^{\prime}\right)\right)=\mathscr{S}(\rho(1, k))$ is in Pos.

It immediately follows from Definition 9 that the prefix-skeleton sampling Pos of (any) trace $\rho$ in an interval $[i, j]$ of $\rho$-positions is such that $|P o s| \leq\left(|S| \cdot 2^{(2 \mid \text { spec } \mid)^{2}}\right)+2$.

Definition 10 ( $h$-prefix sampling). Let $h \geq 0$. The h-prefix sampling of a trace $\rho$ of $\mathcal{K}$ is the minimal set $P S_{h}$ of $\rho$-positions inductively satisfying the following conditions:

- Base case: $h=0 . P S_{0}=\{1,|\rho|\}$;

- Inductive step: $h>0$. (i) $P S_{h} \supseteq P S_{h-1}$ and (ii) for all pairs of consecutive positions $i, j$ in $P S_{h-1}$, the prefix-skeleton sampling of $\rho$ in the interval $[i, j]$ is in $P S_{h}$.

Let $i_{1}<\ldots<i_{N}$ be the ordered sequence of positions in $P S_{h}$ (note that $i_{1}=1$ and $i_{N}=|\rho|$ ). The $h$-sampling word of $\rho$ is the sequence of summaries $\mathscr{S}\left(\rho\left(1, i_{1}\right)\right) \cdots \mathscr{S}\left(\rho\left(1, i_{N}\right)\right)$.

The following upper bound to the cardinality of prefix samplings holds.

Property 11. The $h$-prefix sampling $P S_{h}$ of a trace $\rho$ of $\mathcal{K}$ is such that $\left|P S_{h}\right| \leq\left(|S| \cdot 2^{(2 \mid \text { spec } \mid)^{2}}\right)^{h+1}$.

The following lemma states that, for two traces, the property of having the same $h$-sampling word is a sufficient condition to be $h$-prefix bisimilar.

Lemma 12. For $h \geq 0$, two traces having the same $h$-sampling word are h-prefix bisimilar.

By exploiting the sufficient condition of Lemma 12, we can finally state the exponential-size modeltrace property for $A \bar{A} B \overline{B E}$. In the proof of Theorem 14 below, it is shown how to derive, from any trace $\rho$ of $\mathcal{K}$, an $h$-prefix bisimilar trace $\rho^{\prime}$ induced by $\rho$ (in the sense that $\rho^{\prime}$ is obtained by contracting $\rho$, i.e. by concatenating subtraces of $\rho$ in an ordered way) such that $\left|\rho^{\prime}\right| \leq\left(|S| \cdot 2^{(2 \mid \text { spec } \mid)^{2}}\right)^{h+2}$. By Proposition $8, \rho^{\prime}$ is indistinguishable from $\rho$ w.r.t. the fulfilment of any $\mathrm{A} \overline{\mathrm{A}} \mathrm{B} \overline{\mathrm{B}} \mathrm{E}$ formula $\varphi$ over the set of atomic formulas in spec such that $\mathrm{d}_{\mathrm{B}}(\varphi) \leq h$. We preliminarily define the notion of induced trace (note that if $\pi$ is induced by $\rho$, then $\operatorname{fst}(\pi)=\operatorname{fst}(\rho), \operatorname{lst}(\pi)=\operatorname{lst}(\rho),|\pi| \leq|\rho|$, and $|\pi|=|\rho|$ iff $\pi=\rho)$. 
Definition 13 (Induced trace). Let $\rho$ be a trace of $\mathcal{K}$ of length $n$. A trace induced by $\rho$ is a trace $\pi$ of $\mathcal{K}$ such that there exists an increasing sequence of $\rho$-positions $i_{1}<\ldots<i_{k}$, with $i_{1}=1, i_{k}=n$, and $\pi=\rho\left(i_{1}\right) \cdots \rho\left(i_{k}\right)$.

Theorem 14 (Exponential-size model-trace property for $\mathrm{A} \overline{\mathrm{A}} \overline{\mathrm{B}} \overline{\mathrm{B}})$. Let $\rho$ be a trace of $\mathcal{K}$ and $h \geq 0$. Then there exists a trace $\rho^{\prime}$ induced by $\rho$, whose length is at most $\left(|S| \cdot 2^{(2|s p e c|)^{2}}\right)^{h+2}$, which is h-prefix bisimilar to $\rho$. In particular, for every $\mathrm{A} \overline{\mathrm{A}} \overline{\mathrm{BE}}$ formula $\psi$ with atomic formulas in spec and such that $\mathrm{d}_{\mathrm{B}}(\psi) \leq h$, it holds that $\mathcal{K}, \rho \models \psi$ iff $\mathcal{K}, \rho^{\prime}=\psi$.

Proof. We show that if $|\rho|>\left(|S| \cdot 2^{(2 \mid \text { spec } \mid)^{2}}\right)^{h+2}$, then there exists a trace $\rho^{\prime}$ induced by $\rho$ such that $\left|\rho^{\prime}\right|<|\rho|$ and $\rho$ and $\rho^{\prime}$ have the same $h$-sampling word. Hence, by iterating the reasoning and applying Proposition 8 and Lemma 12 , the thesis follows.

Assume that $|\rho|>\left(|S| \cdot 2^{(2 \mid \text { spec } \mid)^{2}}\right)^{h+2}$. Let $P S_{h}: 1=i_{1}<\ldots<i_{N}=|\rho|$ be the $h$-prefix sampling of $\rho$. By Property 11, $\left|P S_{h}\right| \leq\left(|S| \cdot 2^{(2 \mid \text { spec } \mid)^{2}}\right)^{h+1}$. Since the number of distinct summaries (w.r.t. spec) associated with the prefixes of $\rho$ is at most $|S| \cdot 2^{(2 \mid \text { spec } \mid)^{2}}$, there must be two consecutive positions $i_{j}$ and $i_{j+1}$ in $P S_{h}$ such that for some $\ell, \ell^{\prime} \in\left[i_{j}+1, i_{j+1}-1\right]$ with $\ell<\ell^{\prime}, \mathscr{S}(\rho(1, \ell))=\mathscr{S}\left(\rho\left(1, \ell^{\prime}\right)\right)$. It easily follows that the sequence $\rho^{\prime}$ given by $\rho^{\prime}:=\rho(1, \ell) \cdot \rho\left(\ell^{\prime}+1,|\rho|\right)$ is a trace induced by $\rho$ such that $\left|\rho^{\prime}\right|<|\rho|$ and $\rho$ and $\rho^{\prime}$ have the same $h$-sampling word.

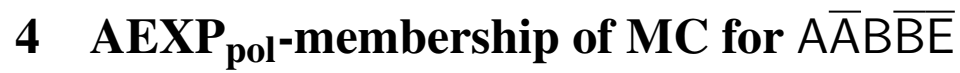

In this section, we exploit the exponential-size model-trace property of $A \bar{A} B \overline{B E}$ to design a MC algorithm for $A \bar{A} B \overline{B E}$ belonging to the class $\mathbf{A E X P}_{\text {pol }}$, namely, the class of problems decidable by singly exponential-time bounded Alternating Turing Machines (ATMs, for short) with a polynomial-bounded number of alternations. More formally, an ATM $\mathscr{M}$ (we refer to [9] or [4] for standard syntax and semantics of ATMs) is singly exponential-time bounded if there is an integer constant $c \geq 1$ such that for each input $\alpha$, any computation starting on $\alpha$ halts after at most $2^{|\alpha|^{c}}$ steps. The ATM $\mathscr{M}$ has a polynomialbounded number of alternations if there is an integer constant $c \geq 1$ such that, for all inputs $\alpha$ and computations $\pi$ starting from $\alpha$, the number of alternations of existential and universal configurations along $\pi$ is at most $|\alpha|^{c}$.

In the sequel, we assume that $\mathrm{A} \overline{\mathrm{A}} \mathrm{B} \overline{\mathrm{B}} \mathrm{E}$ formulas are in $P N F$. For a formula $\varphi$, let spec be the set of regular expressions occurring in $\varphi$. The size $|\varphi|$ of $\varphi$ is given by the number of non-atomic subformulas of $\varphi$, plus $\mid$ spec|. As another complexity measure of an $A \bar{A} B \overline{B E}$ formula $\varphi$, we consider the standard alternation depth, denoted by $\Upsilon(\varphi)$, between the existential $\langle X\rangle$ and universal modalities $[X]$ (and vice versa) occurring in the $P N F$ of $\varphi$, for $X \in\{\bar{B}, \bar{E}\}$. Note that the definition does not consider the modalities associated with the Allen's relations in $\{A, \bar{A}, B\}$. Moreover, let FMC be the set of pairs $(\mathcal{K}, \varphi)$ consisting of a Kripke structure $\mathcal{K}$ and an $\mathrm{A} \overline{\mathrm{A}} \mathrm{B} \overline{\mathrm{BE}}$ formula $\varphi$ such that $\mathcal{K} \models \varphi$. The complexity upper bound is as follows.

Theorem 15. One can construct a singly exponential-time bounded ATM accepting FMC whose number of alternations on an input $(\mathcal{K}, \varphi)$ is at most $\Upsilon(\varphi)+2$.

In the rest of the section, we define a procedure (Figure 11 - which can be easily translated into an ATM - proving the assertion of Theorem 15 . We start with some auxiliary notation. Fix a finite Kripke structure $\mathcal{K}$ with set of states $S$ and an $\bar{A} \overline{\mathrm{BBE}}$ formula $\varphi$ in $P N F$. Let $h=\mathrm{d}_{\mathrm{B}}(\varphi)$, and spec be the set of regular expressions occurring in $\varphi$.

A certificate of $(\mathcal{K}, \varphi)$ is a trace $\rho$ of $\mathcal{K}$ whose length is less than $\left(|S| \cdot 2^{(2 \mid \text { spec } \mid)^{2}}\right)^{h+2}$ (the bound for the exponential trace property in Theorem 14). A $\bar{B}$-witness (resp., $\bar{E}$-witness) of a certificate $\rho$ for 


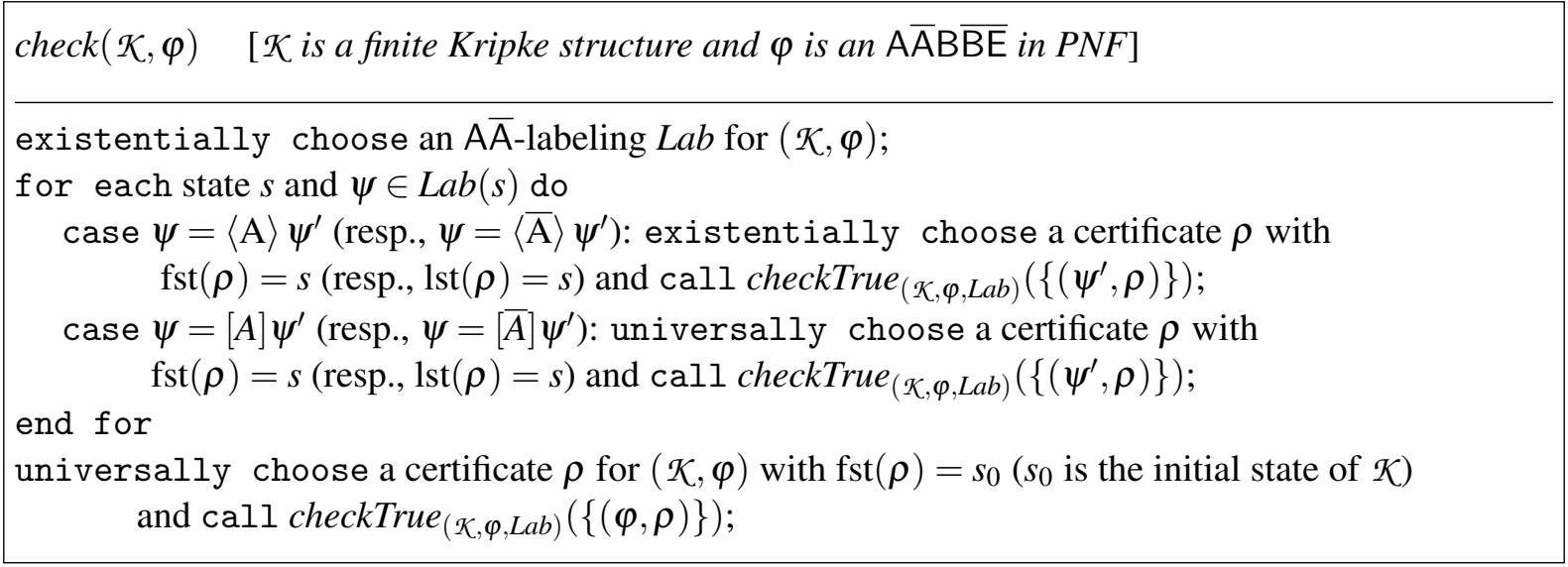

Figure 1: Procedure check

$(\mathcal{K}, \varphi)$ is a certificate $\rho^{\prime}$ of $(\mathcal{K}, \varphi)$ such that $\rho^{\prime}$ is $h$-prefix bisimilar to a trace of the form $\rho \star \rho^{\prime \prime}$ (resp., $\left.\rho^{\prime \prime} \star \rho\right)$ for some certificate $\rho^{\prime \prime}$ of $(\mathcal{K}, \varphi)$ with $\left|\rho^{\prime \prime}\right|>1$. By $\operatorname{SD}(\varphi)$ we denote the set consisting of the subformulas $\psi$ of $\varphi$ and the duals $\widetilde{\psi}$. By the results of Section 3 , we deduce the following:

Proposition 16. Let $\mathcal{K}$ be a finite Kripke structure, $\varphi$ be an $\mathrm{A} \overline{\mathrm{A}} \overline{\mathrm{BE}}$ formula in $P N F$, and $\rho$ be a certificate for $(\mathcal{K}, \varphi)$. The following properties hold:

1. for each $\langle X\rangle \psi \in S D(\varphi)$ with $X \in\{\bar{B}, \bar{E}\}, \mathcal{K}, \rho \models\langle X\rangle \psi$ iff there exists an $X$-witness $\rho^{\prime}$ of $\rho$ for $(\mathcal{K}, \varphi)$ such that $\mathcal{K}, \rho^{\prime} \models \psi$;

2. for each trace of the form $\rho \star \rho^{\prime}$ (resp., $\left.\rho^{\prime} \star \rho\right)$ such that $\rho^{\prime}$ is a certificate for $(\mathcal{K}, \varphi)$, one can construct in time singly exponential in the size of $(\mathcal{K}, \varphi)$, a certificate $\rho^{\prime \prime}$ which is h-prefix bisimilar to $\rho \star \rho^{\prime}$ (resp., $\left.\rho^{\prime} \star \rho\right)$, with $h=\mathrm{d}_{\mathrm{B}}(\varphi)$.

The set $\mathrm{A} \overline{\mathrm{A}}(\varphi)$ is the set of formulas in $\operatorname{SD}(\varphi)$ of the form $\langle X\rangle \psi^{\prime}$ or $[X] \psi^{\prime}$ with $X \in\{A, \bar{A}\}$. An $\mathrm{A} \overline{\mathrm{A}}$-labeling $L a b$ for $(\mathcal{K}, \varphi)$ is a mapping associating to each state $s$ of $\mathcal{K}$ a maximally consistent set of subformulas of $\mathrm{A} \overline{\mathrm{A}}(\varphi)$. More precisely, for all $s \in S, \operatorname{Lab}(s)$ is such that for all $\psi, \widetilde{\psi} \in \mathrm{A} \overline{\mathrm{A}}(\varphi)$, $\operatorname{Lab}(s) \cap\{\psi, \widetilde{\psi}\}$ is a singleton. We say that Lab is valid if for all states $s \in S$ ad $\psi \in \operatorname{Lab}(s), \mathcal{K}, s \models \psi$ (we consider $s$ as a length-1 trace). Finally, a well-formed set for $(\mathcal{K}, \varphi)$ is a finite set $\mathscr{W}$ consisting of pairs $(\psi, \rho)$ such that $\psi \in \operatorname{SD}(\varphi)$ and $\rho$ is a certificate of $(\mathcal{K}, \varphi)$. We say that $\mathscr{W}$ is universal if each formula occurring in $\mathscr{W}$ is of the form $[X] \psi$ with $X \in\{\bar{B}, \bar{E}\}$. The dual $\widetilde{W}$ of $\mathscr{W}$ is the well-formed set obtained by replacing each pair $(\psi, \rho) \in \mathscr{W}$ with $(\widetilde{\psi}, \rho)$. A well-formed set $\mathscr{W}$ is valid if for each $(\psi, \rho) \in \mathscr{W}, \mathcal{K}, \rho \models \psi$.

The procedure check, reported in Figure 1, defines the ATM required to prove the assertion of Theorem 15. The procedure check takes a pair $(\mathcal{K}, \varphi)$ as input and: (1) it guesses an $A \bar{A}$-labeling $L a b$ for $(\mathcal{K}, \varphi) ;(2)$ it checks that the guessed labeling $L a b$ is valid; (3) for every certificate $\rho$ starting from the initial state, it checks that $\mathcal{K}, \rho \models \varphi$. To perform steps (2)-(3), it exploits the auxiliary ATM procedure checkTrue reported in Figure 2. The procedure checkTrue takes as input a well-formed set $\mathscr{W}$ for $(\mathcal{K}, \varphi)$ and, assuming that the current $\mathrm{A} \overline{\mathrm{A}}$-labeling $L a b$ is valid, checks whether $\mathscr{W}$ is valid. For each pair $(\psi, \rho) \in \mathscr{W}$ such that $\psi$ is not of the form $[X] \psi^{\prime}$, with $X \in\{\bar{B}, \bar{E}\}$, checkTrue directly checks whether $\mathcal{K}, \rho \models \psi$. In order to allow a deterministic choice of the current element of the iteration, we assume that the set $\mathscr{W}$ is implemented as an ordered data structure. At each iteration of the while loop in checkTrue, the current pair $(\psi, \rho) \in \mathscr{W}$ is processed according to the semantics of $\mathrm{HS}$, exploiting the guessed $\mathrm{A} \overline{\mathrm{A}}$ labeling $L a b$ and Proposition 16 . The processing is either deterministic or based on an existential choice, 


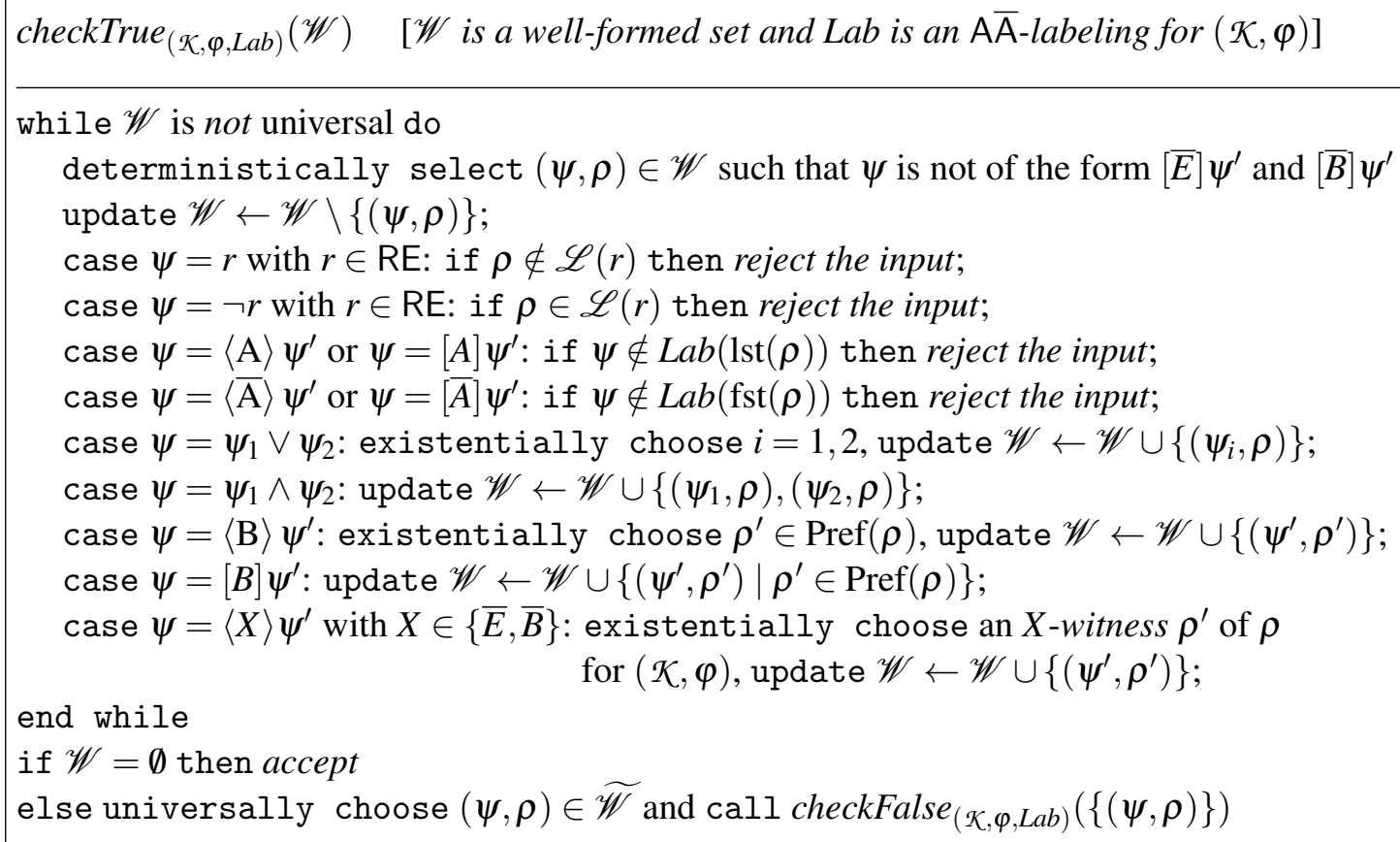

Figure 2: Procedure checkTrue

and the currently processed pair $(\psi, \rho)$ is either removed from $\mathscr{W}$, or replaced with pairs $\left(\psi^{\prime}, \rho^{\prime}\right)$ such that $\psi^{\prime}$ is a strict subformula of $\psi$.

At the end of the while loop, the resulting well formed set $\mathscr{W}$ is either empty or universal. In the former case, the procedure accepts. In the latter case, there is a switch in the current operation mode. For each element $(\psi, \rho)$ in the dual of $\mathscr{W}$ (note that the root modality of $\psi$ is either $\langle\overline{\mathrm{E}}\rangle$ or $\langle\overline{\mathrm{B}}\rangle$ ), the auxiliary ATM procedure checkFalse is invoked, which accepts the input $\{(\psi, \rho)\}$ iff $\mathcal{K}, \rho \not \neq \psi$. The procedure checkFalse is the "dual" of checkTrue: it is simply obtained from checkTrue by switching accept and reject, by switching existential choices and universal choices, and by converting the last call to checkFalse into checkTrue. Thus checkFalse accepts an input $\mathscr{W}$ iff $\mathscr{W}$ is not valid.

Recall that the length of a certificate is singly exponential in the size of the input $(\mathcal{K}, \varphi)$. Thus, since the number of alternations of the ATM check between existential and universal choices is evidently the number of switches between the calls to the procedures checkTrue and checkFalse plus two, by Theorem 14 and Proposition 16, we can state the following result that directly implies Theorem 15 .

Proposition 17. The ATM check is a singly exponential-time bounded ATM accepting FMC whose number of alternations on an input $(\mathcal{K}, \varphi)$ is at most $\Upsilon(\varphi)+2$.

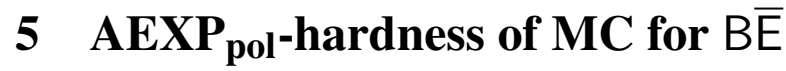

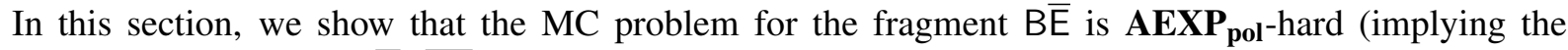
$\left.\mathbf{A E X P}_{\text {pol-hardness of }} A \bar{A} B \overline{B E}\right)$. The result is obtained by a polynomial-time reduction from a variant of the domino-tiling problem for grids with rows and columns of exponential length called alternating multi-tiling problem. 
An instance of this problem is a tuple $\mathscr{I}=\left(n, D, D_{0}, H, V, M, D_{a c c}\right)$, where: $n$ is a positive even natural number encoded in unary; $D$ is a non-empty finite set of domino types; $D_{0} \subseteq D$ is a set of initial domino types; $H \subseteq D \times D$ and $V \subseteq D \times D$ are the horizontal and vertical matching relations, respectively; $M \subseteq D \times D$ is the multi-tiling matching relation; $D_{a c c} \subseteq D$ is a set of accepting domino types. A tiling of $\mathscr{I}$ is a map assigning a domino type to each cell of a $2^{n} \times 2^{n}$ squared grid coherently with the horizontal and vertical matching relations. Formally, a tiling of $\mathscr{I}$ is a mapping $f:\left[0,2^{n}-1\right] \times\left[0,2^{n}-1\right] \rightarrow D$ such that:

- for all $i, j \in\left[0,2^{n}-1\right] \times\left[0,2^{n}-1\right]$ with $j<2^{n}-1,(f(i, j), f(i, j+1)) \in H$;

- for all $i, j \in\left[0,2^{n}-1\right] \times\left[0,2^{n}-1\right]$ with $i<2^{n}-1,(f(i, j), f(i+1, j)) \in V$.

The initial condition Init $(f)$ of the tiling $f$ is the content of the first row of $f$, namely Init $(f):=$ $f(0,0) f(0,1) \ldots f\left(0,2^{n}-1\right)$. A multi-tiling of $\mathscr{I}$ is a tuple $\left(f_{1}, \ldots, f_{n}\right)$ of $n$ tilings which are coherent w.r.t. the multi-tiling matching relation $M$, namely, such that:

- $(i)$ for all $i, j \in\left[0,2^{n}-1\right] \times\left[0,2^{n}-1\right]$ and $\ell \in[1, n-1],\left(f_{\ell}(i, j), f_{\ell+1}(i, j)\right) \in M$ (multi-cell requirement), and (ii) $f_{n}\left(2^{n}-1, j\right) \in D_{a c c}$ for some $j \in\left[0,2^{n}-1\right]$ (acceptance).

The alternating multi-tiling problem for an instance $\mathscr{I}$ is checking whether

- $\forall w_{1} \in\left(D_{0}\right)^{2^{n}}, \exists w_{2} \in\left(D_{0}\right)^{2^{n}}, \ldots, \forall w_{n-1} \in\left(D_{0}\right)^{2^{n}}, \exists w_{n} \in\left(D_{0}\right)^{2^{n}}$ such that there exists a multi-tiling $\left(f_{1}, \ldots, f_{n}\right)$ where for all $i \in[1, n], \operatorname{Init}\left(f_{i}\right)=w_{i}$.

Theorem 18 ([4]). The alternating multi-tiling problem is $\mathbf{A E X P}_{\mathbf{p o l}}$-complete.

The fact that MC for the fragment $B \bar{E}$ is $\mathbf{A E X P}_{\text {pol }}$-hard is an immediate corollary of the following theorem.

Theorem 19. One can construct, in time polynomial in the size of $\mathscr{I}$, a finite Kripke structure $\mathcal{K}_{\mathcal{I}}$ and $a \mathrm{~B} \overline{\mathrm{E}}$ formula $\varphi_{\mathscr{I}}$ over the set of propositions $\mathcal{A P}=D \cup(\{r, c\} \times\{0,1\}) \cup\{\perp$, end $\}$ such that $\mathcal{K}_{\mathscr{I}}=\varphi_{\mathscr{I}}$ iff $\mathscr{I}$ is a positive instance of the alternating multi-tiling problem.

The rest of this section is devoted to the construction of the Kripke structure $\mathcal{K}_{\mathscr{I}}$ and the $\mathrm{B} \overline{\mathrm{E}}$ formula $\varphi_{\mathscr{I}}$, proving Theorem 19 . Let $\mathcal{A P}$ be as in the statement of Theorem 19. The Kripke structure $\mathcal{K}_{\mathscr{I}}$ is given by $\mathcal{K}_{\mathscr{I}}=\left(\mathscr{A P}, S, R, \mu, s_{0}\right)$, where $S=\mathscr{A P}, s_{0}=e n d, \mu$ is the identity mapping (we identify a singleton set $\{p\}$ with $p$ ), and $R=\left\{\left(s, s^{\prime}\right) \mid s \in \mathcal{A P} \backslash\{\right.$ end $\left.\}, s^{\prime} \in \mathcal{A P}\right\}$. Note that the initial state end has no successor, and that a trace of $\mathcal{K}_{\mathscr{G}}$ can be identified with its induced labeling sequence.

The construction of the $\mathrm{B} \overline{\mathrm{E}}$ formula $\varphi_{\mathscr{I}}$ is based on a suitable encoding of multi-tilings which is described in the following. The symbols $\{r\} \times\{0,1\}$ and $\{c\} \times\{0,1\}$ in $\mathscr{A P}$ are used to encode the values of two $n$-bits counters numbering the $2^{n}$ rows and columns, respectively, of a tiling. For a multi-tiling $F=\left(f_{1}, \ldots, f_{n}\right)$ and for all $i, j \in\left[0,2^{n}-1\right]$, the $(i, j)$-th multi-cell $\left(f_{1}(i, j), \ldots, f_{n}(i, j)\right)$ of $F$ is encoded by the word $C$ of length $3 n$ over $\mathcal{A P}$, called multi-cell code, given by $d_{1} \cdots d_{n}\left(r, b_{1}\right) \cdots\left(r, b_{n}\right)\left(c, b_{1}^{\prime}\right) \cdots\left(c, b_{n}^{\prime}\right)$ where $b_{1} \cdots b_{n}$ and $b_{1}^{\prime} \cdots b_{n}^{\prime}$ are the binary encodings of the row number $i$ and column number $j$, respectively, and for all $\ell \in[1, n], d_{\ell}=f_{\ell}(i, j)$ (i.e., the content of the $(i, j)$-th cell of component $f_{\ell}$ ). The content of $C$ is $d_{1} \cdots d_{n}$. Since $F$ is a multi-tiling, the following well-formedness requirement must be satisfied by the encoding $C$ : for all $\ell \in[1, n-1],\left(d_{\ell}, d_{\ell+1}\right) \in M$. We call such words well-formed multi-cell codes.

Definition 20 (Multi-tiling codes). A multi-tiling code is a finite word $w$ over $\mathscr{A P}$ obtained by concatenating well-formed multi-cell codes in such a way that the following conditions hold:

- for all $i, j \in\left[0,2^{n}-1\right]$, there is a multi-cell code in $w$ with row number $i$ and column number $j$ (completeness requirement);

- for all multi-cell codes $C$ and $C^{\prime}$ occurring in $w$, if $C$ and $C^{\prime}$ have the same row number and column number, then $C$ and $C^{\prime}$ have the same content (uniqueness requirement); 
- for all multi-cell codes $C$ and $C^{\prime}$ in $w$ having the same row-number (resp., column number), column numbers (resp., row numbers) $j$ and $j+1$, respectively, and contents $d_{1} \cdots d_{n}$ and $d_{1}^{\prime} \cdots d_{n}^{\prime}$, respectively, it holds that $\left(d_{\ell}, d_{\ell}^{\prime}\right) \in H$ (resp. $\left(d_{\ell}, d_{\ell}^{\prime}\right) \in V$ ) for all $\ell \in[1, n]$ (row-adjacency requirement) (resp., (column-adjacency requirement));

- there is a multi-cell code in $w$ with row-number $2^{n}-1$ whose content is in $D^{n-1} \cdot d_{a c c}$ for some $d_{\text {acc }} \in D_{\text {acc }}$ (acceptance requirement).

Finally, we have to encode the initial conditions of the components of a multi-tiling. An initial cell code encodes a cell of the first row of a tiling and is a word $w$ of length $n+1$ of the form $w=$ $d\left(c, b_{1}\right) \cdots\left(c, b_{n}\right)$, where $d \in D_{0}$ and $b_{1}, \ldots, b_{n} \in\{0,1\}$. We say that $d$ is the content of $w$ and the integer in $\left[0,2^{n}-1\right]$ encoded by $b_{1} \cdots b_{n}$ is the column number of $w$.

Definition 21 (Multi-initialization codes). An initialization code is a finite word $w$ over $\mathcal{A P}$ which is the concatenation of initial cell codes such that:

- for all $i \in\left[0,2^{n}-1\right]$, there is an initial cell code in $w$ with column number $i$.

- for all initial cell codes $C$ and $C^{\prime}$ occurring in $w$, if $C$ and $C^{\prime}$ have the same column number, then $C$ and $C^{\prime}$ have the same content.

A multi-initialization code is a finite word over $\mathscr{A P}$ of the form $\perp \cdot w_{n} \cdots \perp \cdot w_{1} \cdot$ end such that for all $\ell \in[1, n], w_{\ell}$ is an initialization code.

Definition 22 (Initialized multi-tiling codes). An initialized multi-tiling code is a finite word over $\mathcal{A P}$ of the form $\perp \cdot w \cdot \perp \cdot w_{n} \cdots \perp \cdot w_{1} \cdot$ end such that $w$ is a multi-tiling code, $\perp \cdot w_{n} \cdots \perp \cdot w_{1} \cdot$ end is a multiinitialization code, and the following requirement holds:

- for each multi-cell code in $w$ having row number 0 , column number $i$, and content $d_{1} \cdots d_{n}$ and for all $\ell \in[1, n]$, there is an initial cell code in $w_{\ell}$ having column number $i$ and content $d_{\ell}$ (initialization coherence requirement).

We sketch now the idea for the construction of the $B \bar{E}$ formula $\varphi_{\mathscr{I}}$ ensuring that $\mathcal{K}_{\mathscr{I}}=\varphi_{\mathscr{I}}$ iff $\mathscr{I}$ is a positive instance of the alternating multi-tiling problem. We preliminarily observe that since the initial state of $\mathcal{K}_{\mathscr{S}}$ has no successors, the only initial trace of $\mathcal{K}_{\mathscr{I}}$ is the trace end of length 1 . To guess a trace corresponding to an initialized multi-tiling code, $\mathcal{K}_{\mathscr{J}}$ is unraveled backward starting from end, exploiting the modality $\bar{E}$. The structure of the formula $\varphi_{\mathscr{I}}$ is

$$
\varphi_{\mathscr{I}}:=[\bar{E}]\left(\varphi_{1} \rightarrow\langle\overline{\mathrm{E}}\rangle\left(\varphi_{2} \wedge\left(\ldots\left([\bar{E}]\left(\varphi_{n-1} \rightarrow\langle\overline{\mathrm{E}}\rangle\left(\varphi_{n} \wedge\langle\overline{\mathrm{E}}\rangle \varphi_{I M T}\right)\right)\right) \ldots\right)\right)\right) .
$$

The formula $\varphi_{\mathscr{I}}$ features $n+1$ unravelling steps starting from the initial trace end. The first $n$ steps are used to guess a sequence of $n$ initialization codes. Intuitively, each formula $\varphi_{i}$ is used to constrain the $i$-th unravelling to be an initialization code, in such a way that at depth $n$ in the formula a multiinitialization code is under evaluation. The last unravelling step (the innermost in the formula) is used to guess the multi-tiling code. Intuitively, the innermost formula $\varphi_{I M T}$ is evaluated over a trace corresponding to an initialized multi-tiling code, and checks its structure: multi-cell codes are "captured" by regular expressions (encoding in particular their row and column numbers and contents); moreover the completeness, uniqueness, row- and column-adjacency requirements of Definition 20 are enforced by the joint use of $[\bar{E}]$ and regular expressions: intuitively, by means of $[\bar{E}]$, one or two multi-cell codes are generated "separately"; then, if they appear in the considered multi-tiling code, the aforementioned constraints are verified by means of auxiliary formulas, consisting of suitable regular expressions. The initialization coherence requirement of Definition 22 is guaranteed in an analogous way, by comparing initial cell codes and multi-cell codes. Note that the first $n-1$ occurrences of alternations between universal and existential modalities $[\bar{E}]$ and $\langle\overline{\mathrm{E}}\rangle$ correspond to the alternations of universal and existential quantifications in the definition of alternating multi-tiling problem. 
Proposition 23 states the correctness of the construction of $\varphi_{\mathscr{I}}$ (for the definitions of $\varphi_{1}, \ldots, \varphi_{n}$, and $\varphi_{I M T}$, see [4]).

Proposition 23. One can build, in time polynomial in the size of $\mathscr{I}, n+1 \mathrm{~B} \overline{\mathrm{E}}$ formulas $\varphi_{I M T}, \varphi_{1}, \ldots, \varphi_{n}$ such that $\Upsilon\left(\varphi_{I M T}\right)=\Upsilon\left(\varphi_{1}\right)=\ldots=\Upsilon\left(\varphi_{n}\right)=0$, and fulfilling the following conditions.

- For all finite words $\rho$ over $\mathcal{A P}$ of the form $\rho=\rho^{\prime} \cdot \perp \cdot w_{n} \cdots \perp \cdot w_{1} \cdot$ end such that $\rho^{\prime} \neq \varepsilon$ and $\perp \cdot w_{n} \cdots \perp \cdot w_{1} \cdot$ end is a multi-initialization code, $\mathcal{K}_{\mathscr{I}}, \rho \models \varphi_{I M T}$ if and only if $\rho$ is an initialized multi-tiling code.

- For all $\ell \in[1, n]$ and words $\rho$ of the form $\rho=\rho^{\prime} \cdot \perp \cdot w_{\ell-1} \cdots \perp \cdot w_{1} \cdot$ end such that $\rho^{\prime} \neq \varepsilon$ and $w_{j} \in(\mathscr{A P} \backslash\{\perp\})^{*}$ for all $j \in[1, \ell-1], \mathcal{K}_{\mathcal{I}}, \rho=\varphi_{\ell}$ if and only if $\rho^{\prime}$ is of the form $\rho^{\prime}=\perp \cdot w_{\ell}$, where $w_{\ell}$ is an initialization code.

Since the initial state of $\mathcal{K}_{\mathscr{g}}$ has no successors and corresponds to the atomic proposition end, by Proposition 23 and Definitions 20 22, we obtain that $\mathcal{K}_{\mathscr{I}} \models \varphi_{\mathscr{I}}$ iff $\mathscr{I}$ is a positive instance of the alternating multi-tiling problem. This concludes the proof of Theorem 19.

\section{Conclusions and future work}

In this paper, we have investigated the MC problem for two maximal fragments of $H S, A \bar{A} B \overline{B E}$ and $A \bar{A} E \overline{B E}$, endowed with interval labeling based on regular expressions, and we have proved that such

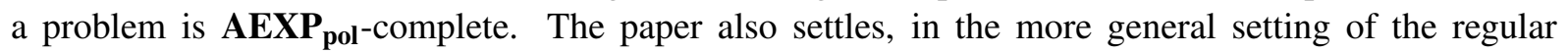
expression-based semantics, the open complexity question for the same fragments under the homogeneity assumption. Future work will focus on the problem of determining the exact complexity of MC for full HS, both under homogeneity and in the regular expression-based semantics. In addition, we will study the MC problem for HS over visibly pushdown systems (VPS), in order to deal with recursive programs and infinite state systems. Finally, we are thinking of inherently interval-based models of systems. Kripke structures, being based on states, are naturally oriented to the description of point-based properties of systems, and of how they evolve state-by-state. We want to come up with suitable (and practical) description paradigms for systems, which allow us to directly model them on the basis of their interval behavior/properties. Only after devising these models (something that seems to be extremely challenging), a really general interval-based $\mathrm{MC}$ will be possible.

\section{References}

[1] J. F. Allen (1983): Maintaining Knowledge about Temporal Intervals. Communications of the ACM 26(11), pp. 832-843, doi $10.1145 / 182.358434$.

[2] L. Bozzelli, H. van Ditmarsch \& S. Pinchinat (2015): The Complexity of One-agent Refinement Modal Logic. Theoretical Computer Science 603(C), pp. 58-83, doi $10.1016 / j . t c s .2015 .07 .015$.

[3] L. Bozzelli, A. Molinari, A. Montanari \& A. Peron (2017): An in-Depth Investigation of Interval Temporal Logic Model Checking with Regular Expressions. In: SEFM. Available at https://www.dimi.uniud.it/ la-ricerca/pubblicazioni/preprints/2.2017/.

[4] L. Bozzelli, A. Molinari, A. Montanari \& A. Peron (2017): On the Complexity of Model Checking for Syntactically Maximal Fragments of the Interval Temporal Logic HS with Regular Expressions. Technical Report, University of Udine, Italy. Available at https://www.dimi.uniud.it/la-ricerca/pubblicazioni/ preprints/3.2017/

[5] L. Bozzelli, A. Molinari, A. Montanari, A. Peron \& P. Sala (2016): Interval Temporal Logic Model Checking: the Border Between Good and Bad HS Fragments. In: IJCAR, LNAI 9706, pp. 389-405, doi:10.1007/9783-319-40229-1_27. 
[6] L. Bozzelli, A. Molinari, A. Montanari, A. Peron \& P. Sala (2016): Interval vs. Point Temporal Logic Model Checking: an Expressiveness Comparison. In: FSTTCS, pp. 26:1-14, doi 10.4230/LIPIcs.FSTTCS.2016.26.

[7] L. Bozzelli, A. Molinari, A. Montanari, A. Peron \& P. Sala (2016): Model Checking the Logic of Allen's Relations Meets and Started-by is $\mathbf{P}^{\mathbf{N P}}$-Complete. In: GandALF, pp. 76-90, doi 10.4204/EPTCS.226.6

[8] D. Bresolin, D. Della Monica, V. Goranko, A. Montanari \& G. Sciavicco (2014): The dark side of interval temporal logic: marking the undecidability border. Annals of Mathematics and Artificial Intelligence 71(13), pp. 41-83, doi:10.1007/s10472-013-9376-4.

[9] A. K. Chandra, D. C. Kozen \& L. J. Stockmeyer (1981): Alternation. Journal of the ACM 28(1), pp. 114-133, doi: $10.1145 / 322234.322243$.

[10] E. A. Emerson \& J. Y. Halpern (1986): "Sometimes" and "not never" revisited: on branching versus linear time temporal logic. Journal of the ACM 33(1), pp. 151-178, doi:10.1145/4904.4999.

[11] J. Ferrante \& C. Rackoff (1975): A Decision Procedure for the First Order Theory of Real Addition with Order. SIAM Journal of Computation 4(1), pp. 69-76, doi:10.1137/0204006.

[12] F. Giunchiglia \& P. Traverso (1999): Planning as Model Checking. In: ECP, LNCS 1809, Springer, pp. 1-20, doi $10.1007 / 10720246 \_1$

[13] J. Y. Halpern \& Y. Shoham (1991): A Propositional Modal Logic of Time Intervals. Journal of the ACM 38(4), pp. 935-962, doi:10.1145/115234.115351.

[14] S. C. Kleene (1956): Representation of Events in Nerve Nets and Finite Automata. In: Automata Studies, 34, Princeton University Press, pp. 3-41.

[15] A. Lomuscio \& J. Michaliszyn (2013): An Epistemic Halpern-Shoham Logic. In: IJCAI, pp. 1010-1016. Available at http://dl.acm.org/citation.cfm?id=2540128.2540274.

[16] A. Lomuscio \& J. Michaliszyn (2014): Decidability of model checking multi-agent systems against a class of EHS specifications. In: ECAI, pp. 543-548, doi:10.3233/978-1-61499-419-0-543

[17] A. Lomuscio \& J. Michaliszyn (2016): Model Checking Multi-agent Systems Against Epistemic HS Specifications with Regular Expressions. In: KR, AAAI Press, pp. 298-307.

[18] A. Lomuscio \& F. Raimondi (2006): MCMAS: A Model Checker for Multi-agent Systems. In: TACAS, LNCS 3920, Springer, pp. 450-454, doi:10.1007/11691372_31.

[19] J. Marcinkowski \& J. Michaliszyn (2014): The Undecidability of the Logic of Subintervals. Fundamenta Informaticae 131(2), pp. 217-240, doi 10.3233/FI-2014-1011.

[20] A. Molinari, A. Montanari, A. Murano, G. Perelli \& A. Peron (2016): Checking interval properties of computations. Acta Informatica, doi:10.1007/s00236-015-0250-1.

[21] A. Molinari, A. Montanari \& A. Peron (2015): Complexity of ITL model checking: some well-behaved fragments of the interval logic HS. In: TIME, pp. 90-100, doi:10.1109/TIME.2015.12.

[22] A. Molinari, A. Montanari \& A. Peron (2015): A Model Checking Procedure for Interval Temporal Logics based on Track Representatives. In: CSL, pp. 193-210, doi 10.4230/LIPIcs.CSL.2015.193.

[23] A. Molinari, A. Montanari, A. Peron \& P. Sala (2016): Model Checking Well-Behaved Fragments of HS: the (Almost) Final Picture. In: KR, pp. 473-483.

[24] B. Moszkowski (1983): Reasoning About Digital Circuits. Ph.D. thesis, Stanford University, CA.

[25] A. Pnueli (1977): The temporal logic of programs. In: FOCS, IEEE, pp. 46-57, doi 10.1109/SFCS.1977.32.

[26] I. Pratt-Hartmann (2005): Temporal prepositions and their logic. Artificial Intelligence 166(1-2), pp. 1-36, doi $10.1016 /$ j.artint.2005.04.003

[27] P. Roeper (1980): Intervals and Tenses. J. Philosophical Logic 9, pp. 451-469, doi 10.1007/BF00262866.

[28] Y. Venema (1990): Expressiveness and Completeness of an Interval Tense Logic. Notre Dame Journal of Formal Logic 31(4), pp. 529-547, doi:10.1305/ndjfl/1093635589 